\title{
Integration of BIM and Computer Simulations in Modular Construction, A Case Study
}

\author{
Wu Chengke ${ }^{1}$, Jiang Rui ${ }^{2}$, Li Xiao ${ }^{3}$ \\ ${ }^{1}$ M.S., Faculty of Construction Management and Real Estate, Chongqing University \\ ${ }^{2}$ M.S., Faculty of Construction Management and Real Estate, Chongqing University \\ ${ }^{3}$ PhD candidate, The Hong Kong Polytechnic University
}

\begin{abstract}
:
Construction Sector has long been criticized for its lower productivity compared with other industries. To address the problem, recent years, new construction methods and information techniques such as modular construction and Building Information Modelling (BIM) are developed and implemented. Besides, computer simulations, like Discrete Event Simulation (DES) and Agent Based Simulation (ABS), are also incorporated in construction sector. Despite contributions of the 3 techniques to the industry have been investigated respectively by many researches, more benefits could be brought if they are applied simultaneously. However, there is no comprehensive research yet to combine all of them in a single project. To bridge the research gap, this paper first briefly introduces strengths and current applications of the 3 techniques; then a framework integrating them together containing simulation module, BIM database module and decision making module is constructed; finally, a case study of a multistoried canteen project is demonstrated to further explain functions of the framework.
\end{abstract}

\section{Introduction}

Architecture Engineering and Construction (AEC) sector has long been criticized for low productivity, damages to environment and lack of innovations ${ }^{[1]}$. To address these problems, on the one hand, modular construction has been extensively implemented due to higher construction speed and less pollutions without sacrificing quality; on the other hand, various Information Technologies have been adopted, among which BIM is recognized as the most useful one. Besides, along with fast developments of computer science, computer simulation has become a hot topic in the industry. With the computing power, complicated interactions of construction process can be represented virtually, considering numerous variables, statistical distributions and uncertainties to facilitate informed decision making, which is hardly achieved in ordinary project management software ${ }^{[2]}$. Due to enormous benefits, lots of scholars have done in-depth studies on these techniques. However, comprehensive studies revealing mutual effects and working processes when Modular Construction Method, BIM and Computer Simulation are combined in integrated environment are yet conducted. To bridge the knowledge gap, this paper first briefly introduces key features and current applications of the 3 techniques; then a framework integrating them together is developed and discussed; finally, a restaurant project is analyzed to better understand the effects of different delivery schedule on cost.

\section{Characteristics and Values of Modular Construction, BIM and Computer Simulations}

Modular construction has experienced decades of development and is still playing an important 
role today. In modular construction most components are all manufactured in factories as modules then transported to construction site to be assembled. Main values and benefits of modular construction can be divided into 6 main categories: 1) Cost saving because of scale of economy in factory manufacturing, less auxiliary equipment and minimized design errors; 2) Schedule compression, realized by fast on-site assembling process and less conflicts and reworks; 3) Increased financial returns since projects are completed earlier for sale; 4) Quality improvements supported by standardized manufacturing and assembling procedures; 5) Environment protected enabled by less wet work conducted on site; and 6) Safety enhancement due to both workload and labor are less required ${ }^{[3]}$.

BIM is recognized as a digital representation of building, comprising rich information which can be used for different purposes covering whole project lifecycle. The powerful data storing and extracting capacities of BIM provide project practitioners with an effective communication platform, which well satisfies strict information requirements of coordination in projects ${ }^{[4]}$. However, for one thing, many types of uncertainties and randomness, which may dramatically impact performance of real projects, are not sufficiently addressed in most BIM applications; for another, BIM focus on physical building analysis while to some degree ignore unphysical factors like human behaviors ${ }^{[5]}$.

Computer simulation is particularly useful when objects or processes in question 1) are highly uncertain and multiple probability distributions need to be considered; 2) are affected by complicated interactions of both internal and external elements; 3) dynamic outcomes are required but are difficult to predict. Among all simulation models, DES and ABS are the most extensively recognized ones in construction. DES model consists of entities, resources and activities, where entities go through activities sequentially and consume resources. By setting capacity of resources, consumption rates of entities and parameters of relating activities, one can monitor and test performance of the process. Thus DES is particularly useful to solve construction problem of sequential nature, like onsite concrete works and factory production. However, conditions and interactions of individual elements are generally ignored in DES. On the other hand, ABS model is constructed by a set of artificial agents which are able to sense and stochastically respond to local environments to dynamically evolve and make autonomous decisions. Characteristics of ABS include: 1) Each type of agent may have unique and diverse parameters, variables and rules; 2) agents interact with each other at micro level, but will determine complex system behaviors at macro level; 3) there is usually no central control system in $\mathrm{ABS}^{[6]}$. ABS significantly increases flexibility and reliability of project management from a decentralized prospective, concentrating on individual elements, such as workers, to address inherent unpredictability, which is hardly achieved in DES model ${ }^{[7]}$.

\section{Current Applications of BIM and Computer Simulations in Modular Construction}

Accurate and timely engineering information synchronization is crucial for satisfactory outcomes in modular construction. Thus, BIM is developed as a platform, allowing project practitioners working on the same integrated BIM model, where construction information is created, processed and exchanged in a cooperative manner. BIM contributes to modular construction mostly in 3 aspects: 1) communicates any changes during construction in real time 
to balance manufacturing progress in factory, addressing graphical separation problem between the 2 sites. 2) runs clash detections, especially for MEP systems installation, which are quite error prone without BIM. 3) corrects design errors visually with standardized manufacturing procedures in $3 \mathrm{D}$ models to avoid serious ripple effects in subsequent process ${ }^{[8]}$.

Given strengths of DES, it is extensively adopted in optimizing components production cycle and managing onsite inventory to balance manufacturing and assembling ${ }^{[9]}$. In contrast, ABS is applied more in construction and operation phases to solve problems involving human behaviors, safety culture, morale, organizational relationships and supply chain ${ }^{[6]}$. Provided strict requirements of data reliability and timeless, BIM is commonly used as a central information repository for simulations.

In summary, BIM, DES and ABS all have distinct advantages. Unfortunately, till now DES is the only simulation tool in studies integrating computer simulations and BIM in modular construction. However, ABS should also be integrated to flexibly manage individual construction elements to shed lights on factors ignored in DES model. Therefore, in following sections, a framework combining BIM, DES and ABS in modular construction is developed.

\section{Methodology}

The framework is formed by 3 modules: BIM database module, Computer Simulation Module and Decision Making Module. Structure and work flow in the framework is shown in Fig. 1.

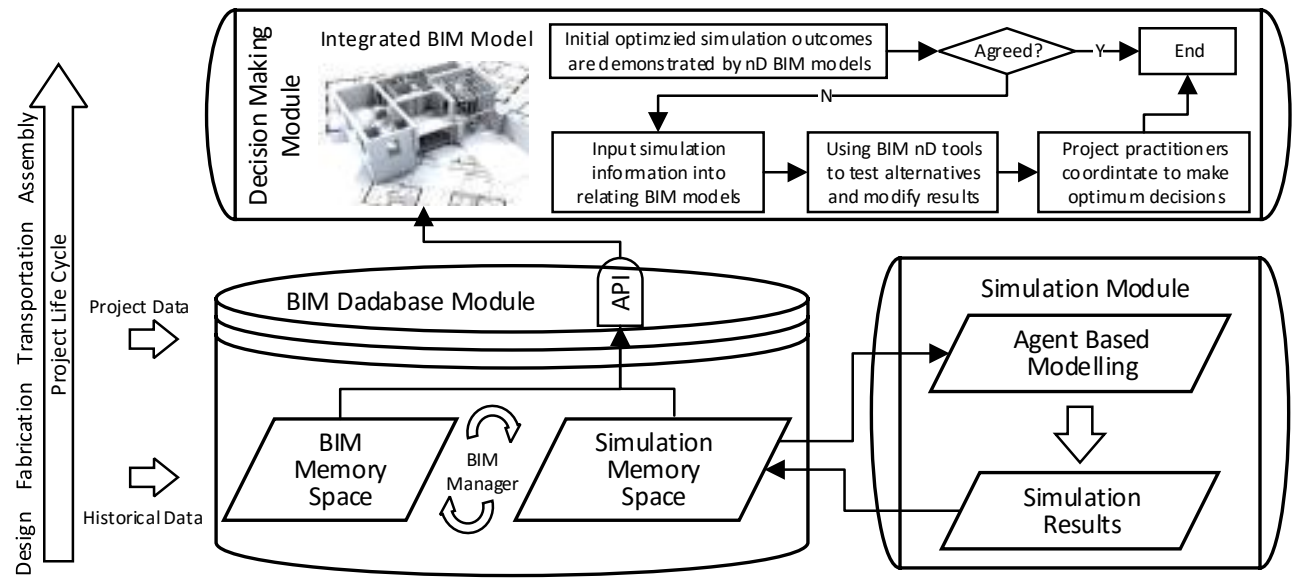

Fig. 1 Structure and Workflow of Proposed Framework

\section{BIM Database Module}

In projects applying BIM, based on the fundamental BIM model provided by design company, practitioners of different disciplines construct and maintain their own BIM models for specialized analysis then coordinate with others. During the process, data and information of both non-graphical and graphical types from all practitioners are stored in BIM database for project monitoring, control, profits distributions, etc. Therefore, in the proposed framework, BIM database is regarded as the information source. To take fully use of existing project BIM database, a new simulation memory space (SMS) is specially developed within the database for simulation purposes. In SMS, simulation relating information is classified and stored while XML or XLS format files are prepared for externally accesses from simulation models, 
avoiding conflictions with original BIM memory spaces (BMS). However, internal information exchange between SMS and BMS is bidirectional, which on the one hand allows information stored in BMS to be directly extracted into SMS; on the other hand, simulation results may be fed back to BMS to improve performance of BIM tools, see Fig. 3.

\section{Simulation Module}

Simulation module is the core in the proposed framework, formed by DES based manufacturing model and ABS based onsite model. Following contents introduce some basic model elements in modular construction, however, it is critical to modify detail settings for specific purposes.

\section{(1) Factory Manufacturing Model}

DES model is used to simulate manufacture of building components. For simplification, the model only considers 3 fundamental procedures, namely framing, pouring and sheathing, where production durations are controlled by statistic distributions based on historical data. Besides, prepared components are directly linked to onsite ABS model to take strengths of both simulation techniques meanwhile achieve simultaneous information transmission, see Figure 2.

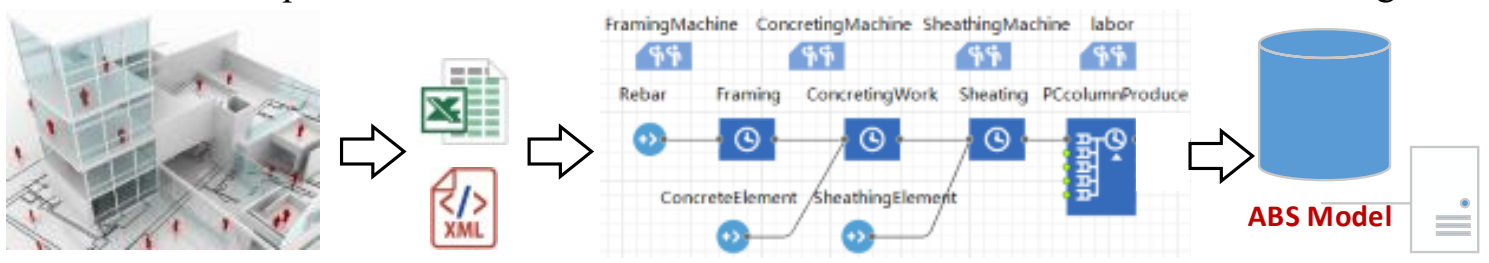

Fig. 2 Information Flow between BIM Model and DES Model

\section{(2) On Site Construction Model}

Every type of agent consists of several elements, including parameters, state-charts, transitions and events etc. Basic introductions of these ABS elements are summarized in Table 2.

Table 2: Introduction of ABS elements

\begin{tabular}{cl}
\hline Name & \multicolumn{1}{c}{ Descriptions } \\
\hline \multirow{5}{*}{ States } & $\begin{array}{l}\text { States are used to define the process that agents may go through. However, } \\
\text { unlike unidirectional processes in most DES model, states changes of ABS in } \\
\text { many cases are multidirectional and iterative, controlled not only by resources } \\
\text { availability but also by extensive unphysical factors. One state could be linked } \\
\text { to various other states, representing different consequences of agents } \\
\text { interactions and finally form a "state-chart". }\end{array}$ \\
Change of states are triggered by 5 types of transitions: Timeout, Rate, \\
Transitions \\
$\begin{array}{l}\text { Condition, Agents Arrivals and Message, each of which is controlled by either } \\
\text { stochastic or deterministic values. There are also internal transitions which only } \\
\text { happens among agents in particular state to represent repetitive actions. }\end{array}$ \\
Parameters & $\begin{array}{l}\text { Parameters are attributes of agents thus do not vary during simulation. It is } \\
\text { worth to note that parameters are commonly used as transition values and they } \\
\text { can also be interactive for adjustments to dynamically control simulation } \\
\text { Event is particular flexible element because they are defined outside of state- } \\
\text { chart thus could affect different types of agents in any state. }\end{array}$
\end{tabular}

Considering characteristics of modular construction, there are total 3 major types of agents, 1) building components, including beams, columns, panelized walls, etc.; 2) onsite equipment, such as cranes; and 3) assembling labors. Table 3-4 and Figure 4-6 summarizes their formations. 


\section{- Building Components}

Based on ordinary procedures in modular construction, 6 states for most types of building components to go through before they are assembled.

Table 3: State-chart of Building Components Agents

\begin{tabular}{|c|c|}
\hline Name & Descriptions \\
\hline InFactory: & $\begin{array}{l}\text { In this state, building components are being transported to construction site. } \\
\text { Transition is set to be Message, depending on transportation schedule. }\end{array}$ \\
\hline EntryCheck & $\begin{array}{l}\text { Building components are checked before stored. Transition of this state } \\
\text { contains a branch, where defective components go to "Repair" state while } \\
\text { the others go to "Storage" State waiting for installation. }\end{array}$ \\
\hline Repair & $\begin{array}{l}\text { Defective components need to be repaired and go back to "onSiteStorage" } \\
\text { state through "Timeout" transition. The internal transition in this state is to } \\
\text { calculate Repair costs. }\end{array}$ \\
\hline OnSiteStorage & $\begin{array}{l}\text { This state represents onsite modular construction inventory and have direct } \\
\text { influence on project costs. } 2 \text { internal transitions are inserted, one is for } \\
\text { building components to request resources availability information by } \\
\text { iterating labors and equipment; while another is for recording cost, both of } \\
\text { them are triggered by a given "Rate". }\end{array}$ \\
\hline Installation & $\begin{array}{l}\text { To enter "Installation" state, building components have to receive a } \\
\text { "message" from labors which indicate their availability, then components } \\
\text { will be connected to those labors and installation will be carried out. }\end{array}$ \\
\hline Installed & $\begin{array}{l}\text { ter the labors have finished the assembling, a message is sent to building } \\
\text { mponents which will trigger the final transition to "Installed" }\end{array}$ \\
\hline
\end{tabular}

The state-chart needs 6 parameters: 1) 3 cost relating parameters to calculate manufacturing, assembling and storage cost respectively; 2) "DefectRate" to determine percentage of components identified as defective; 3) "TimeForRepair" to control necessary repair time.

- Labors

Labors are responsible for onsite assembling and 3 states are included in this agent type.

Table 4: State-chart of Labor agents

\begin{tabular}{cl}
\hline Name & \multicolumn{1}{c}{ Descriptions } \\
\hline Idle & $\begin{array}{l}\text { In this state, labors are waiting for building components in storage to send them a } \\
\text { message to start assembling process. } \\
\text { Labor will become "Busy" if particular message is received. In this stage, labors can } \\
\text { either finish their job after a certain duration then go back to "Idle", or get injured and }\end{array}$ \\
gusy to "MinorInjury" state. The former is triggered by "Timeout" representing \\
production rates; the latter is controlled by "Rate", which shall comply with accident \\
rates in real projects. \\
Labors are unable to process works if they are in "Injury" State and have to wait until \\
fully recover, the transition is "Timeout", which refers to minor accidents.
\end{tabular}

Parameters in the state-chart are mainly embedded in "Busy" to "Idle" transition, including factors which affect production rates, such as morale, weather changes, fatigue, etc.;

\section{- Equipment}

Equipment state-chart is similar with Labors but is simpler since only "Idle" and "Working" states are developed. "WeatherEffects" and "ProductionRates" are the 2 major parameters in this agent type, which reveal environment influnces and lifting speed respectively. 


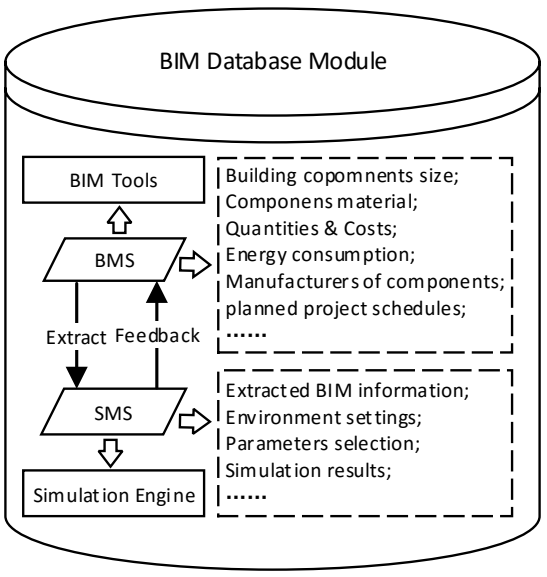

Fig. 3 Structure of BIM Database Module

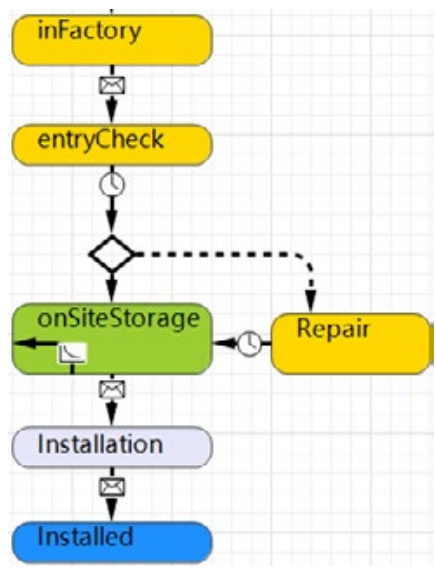

Fig. 4 Component state-chart

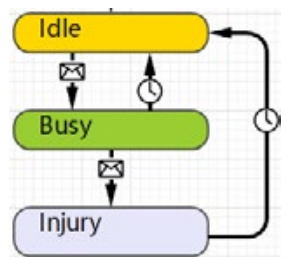

Fig.5 Labor state-chart

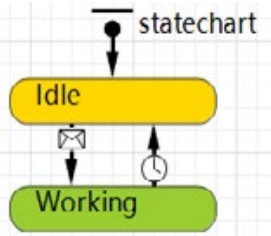

Fig. 6 Equip state-chart

\section{Decision Making Module}

Results from simulation models are usually abstract and confusing for stakeholders. However, BIM tools could provide project documents, including plans, sections, MEP fixtures, shop drawings, etc. while running various analysis in visualized manner. Therefore, Decisions Making Module utilizes BIM to explain simulation results and facilitate informed decision making. For instance, assembling sequences have been exported by ABS. However, owner, contractor and manufacturer, etc. still need to determine which one to be adopted or if modification is necessary based on their own demands. Thus simulation results are fed back to BIM models to carry out $\mathrm{nD}$ analysis, where original sequences are compared and adjusted in cooperative environment to yield decisions satisfying requirements of each player.

\section{Case Study}

The case study is a canteen project in Chongqing, China. Audodesk Revit 2015 is the major tool to develop architecture and structural BIM models, which are shown in Fig. 7. To comply with local construction regulations, LuBan BIM Software is used to input IFC files from Revit to insert rebar, materials and other built-in elements. Then, XML and XLS files are generated from Luban and Revit database and sent to simulation engines built in AnyLogic version 7.1.

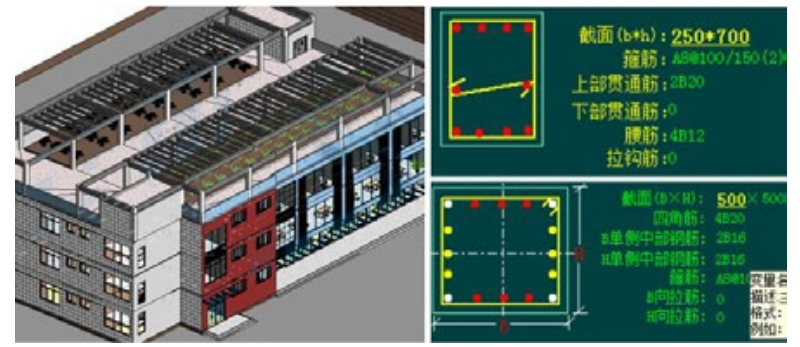

Fig. 7 Column and Beam in BIM Model

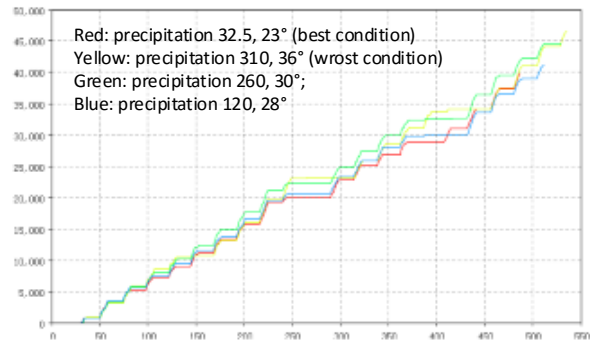

Fig. 8 Effects of Weather and Morale

Columns and beams with simplified sizes of first floor are the representative components because they are enough to represent basic assembling sequence taking construction sections into account, while keep the case model simple. Besides, crane is the only onsite equipment to lift building components. The main purpose of simulation is to determine delivery schedule while consider weather effects and labor morale. The onsite contractor first tries different delivery plans with given labor resources, then the chosen plan is communicated with 
manufacturer for modifications. Required data and information flow are shown in Fig. 9.

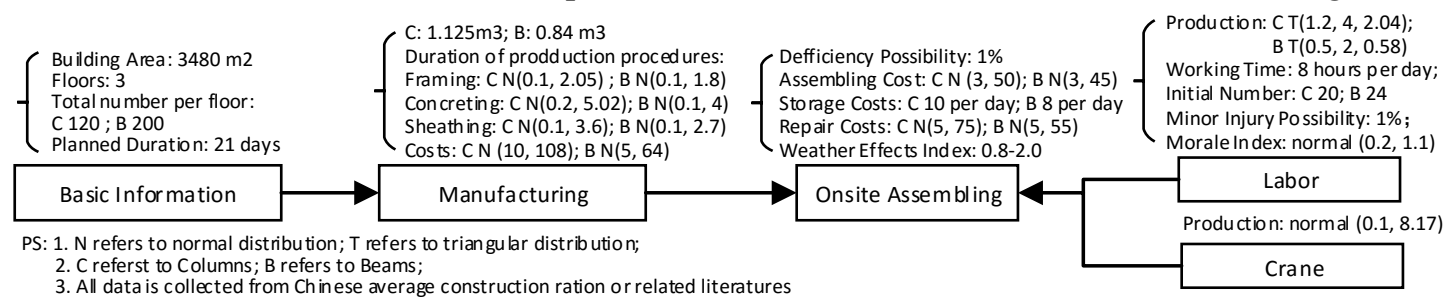

Fig. 9 Information Flow in Canteen Case

3 plans are tested: 1) delivering all components one time; 2) delivering components based on construction section division; 3) delivering certain number of components at given interval. Fig. 10 shows costs distribution of 3 scenarios. It is obvious that one-off plan causes unnecessary storage fees which increases costs by $49.9 \%$; while continuous plan seriously delays project completion by $194 \%$. It seems construction section-based plan is the optimum one since it reduces cost by $19.5 \%$, while keeps original schedule of 21 days unaffected. Hence, assembling is planned to begin at $1^{\text {st }}$ and end at $19^{\text {th }}$ April. However, the commencement of construction is assumed to be delayed, which not only contradicts production plan with manufacturer but also makes section-based transportation impossible. Besides, the delay will negatively affect assembling because of rainy season and morale. After communication with manufacturer and owner using BIM 4D scheduling and 5D cost estimation, the contractor rearranges the schedule to be continuous type with 20 columns delivered each time. Weather and morale effects in the adopted plan, based on historical data and existing survey ${ }^{[10]}$ is shown in Fig. 8. It should be note that cost and duration do not vary much only because the construction scale is small. However, these factors still need to be carefully controlled in subsequent works.
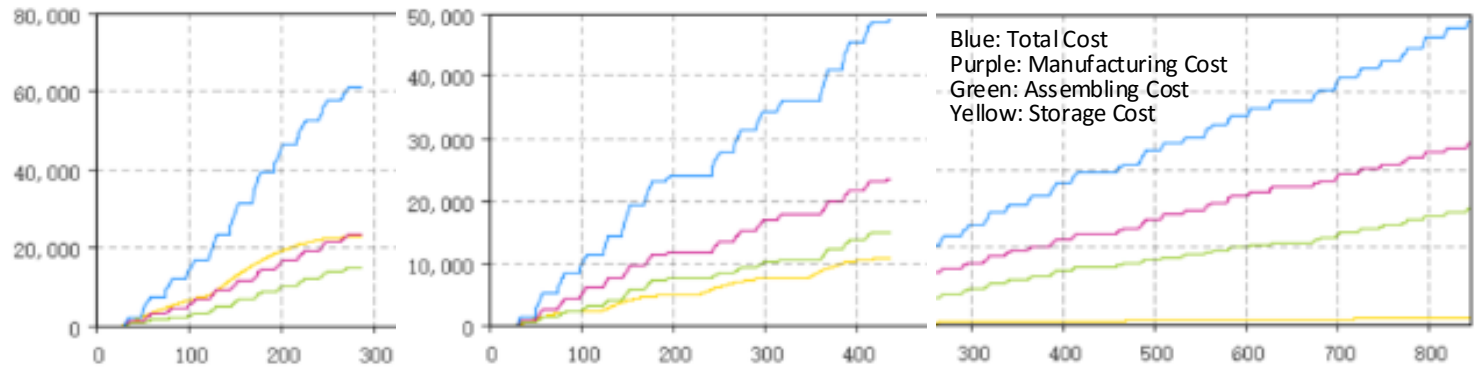

Fig. 10 Cost Comparisons and Distributions of Different Transportation Plans

\section{Discussion}

There are 3 major features of the proposed framework: 1) ABS and DES are simultaneously implemented in modular construction, where more types of influencing factors are involved and advantages of 2 techniques could be taken to meet requirements in both factory and assembling sites. 2) BIM is used as a demonstration and decision making tool, while remains to be the information source, thus any changes can be communicated and modified timelessly, closely linking framework modules and significantly increases project coordination level. 3) The framework is highly flexible to accommodate multiple types of simulation models and solve problems of different complicity, according to specific needs of project practitioners. However, there are some limitations need to be addressed: 1) Many influencing factors existing in modular construction environment, such as learning curve, equipment breakdown, labor 
fatigue, etc. are still ignored. 2) Only most basic manufacturing and assembling process is simulated in case study, which is unable to represent construction logics in real projects. However, fundamental concepts and principles of the proposed framework have been demonstrated. Besides, as mentioned, further modifications of the framework should be determined at project level to match projects' features. Therefore, it is necessary to implement the framework in ongoing modular construction projects to test its feasibility in the future.

\section{Conclusion}

This paper develops a framework that integrates BIM, DES and ABS in modular construction. This framework on the one hand has shown that BIM could 1) be an effective source to provide information for DES and ABS models 2) receive feedbacks from simulation models to update and optimize original data; 3 ) communicate results among stakeholders to realize timeless coordination. On the other hand, it takes fully use of computer simulations to address complex interactions to manage onsite and offsite works and support decision-making, where roles of different types of computer simulations are discussed in detail. In conclusion, BIM, DES and ABS could complement each other to improve performance of modular construction projects.

\section{Acknowledgement}

We gratefully acknowledge the financial support from Chongqing Graduate Student Research Innovation Project (CYS14036)

\section{Reference}

[1] Liang Xiao, Liu Yanling (2013), The development roadmap of BIM in Chinese Building Industry [C], 15th annual meeting of science and technology, China.

[2] AbouRizk S. Role of simulation in construction engineering and management[J]. Journal of construction engineering and management, 2010, 136(10): 1140-1153.

[3] Rogan A L, Lawson R M, Bates-Brkljac N. Value and benefits assessment of modular construction[J]. The Steel Construction Institute, Ascot, 2000.

[4] Barlish K, Sullivan K. How to measure the benefits of BIM-A case study approach[J]. Automation in construction, 2012, 24: 149-159.

[5] Liu H, Al-Hussein M, Lu M. BIM-based integrated approach for detailed construction scheduling under resource constraints [J]. Automation in Construction, 2015, 53: 29-43.

[6] Sawhney A, Bashford H, Walsh K, et al. Agent-based modeling and simulation in construction[C]//Simulation Conference, 2003. Proceedings of the 2003 Winter. IEEE, 2003, 2: 1541-1547.

[7] Maidstone R. Discrete event simulation, system dynamics and agent based simulation: Discussion and comparison[J]. System, 2012: 1-6.

[8] Lu N, Korman T. Implementation of building information modeling (BIM) in modular construction: Benefits and challenges[C]/Proceedings of the Construction Research Congress, Banff, Alta. 2010: 8-10.

[9] Liu H, Altaf M S, Lei Z, et al. Automated production planning in panelized construction enabled by integrating discrete-event simulation and BIM[J]. 2015.

[10] Birgisson K. Discrete-Event Simulations of Construction Related Production System[J]. TVBK, 2009. 